\title{
Non-invasive 3D Crystallography of Geological Media in the Laboratory
}

Matthew Andrew ${ }^{1 *}$, Hrishikesh Bale ${ }^{1}$, Nicolas Gueninchault ${ }^{1}$, Jun Sun $^{1}$, Romy Hanna ${ }^{2}$, Jessica

Maisano $^{2}$, Richard Ketcham ${ }^{2}$, Matthew Pankhurst ${ }^{4}$ and Mike Zolensky ${ }^{3}$

1. Carl Zeiss X-ray Microscopy, Pleasanton, CA, USA.

2. Jackson School of Geosciences, University of Texas, Austin, TX, USA.

3. ARES, NASA JSC, Houston, TX, USA.

4. ITER, 38600 Granadilla de Abona, Tenerife, Canary.

* Corresponding author: matthew.andrew@zeiss.com

One of the main challenges in geological microstructural analysis is to understand the details and distribution of the mineral crystals from which the rock is constructed, principally investigated using Electron Back Scatter Diffraction (EBSD) [1]. Although this technique has provided great insight into rock deformation, metamorphic processes, planetary geology and geochronology it suffers from the inherent limitations that it can only be performed on the surface of a polished sample under the vacuum conditions of an electron microscope. And while it is possible to extend this technique into 3D by coupling with Focused Ion Beam (FIB) serial sectioning, this is destructive, extremely slow and not practical for most applications. It also does not allow for samples to be subjected to experimental loading, temperature or deformation which would allow for the direct investigation of geological processes in situ.

Recent years have seen the development of Diffraction Contrast Tomography (DCT), initially using monochromatic X-ray beams found at 3rd generation synchrotron light sources [2], and more recently adapted to polychromatic sources of laboratory X-ray microscopes (referred to as LabDCT [3]). However, examination to date has been limited to the investigation of systems of relative crystallographic simplicity, made of large, well-formed crystals of high crystal symmetry. Most geological minerals have a much lower order symmetry, and may be deformed, heavily twinned, degraded, or subject to a range of other mineralogical defects which make their examination consequentially more challenging. In this study we show the results from this technique as applied to three systems - the San Carlos Olivine and two meteorite chondrules (a porphyritic olivine and a barred olivine). All data were acquired using the Versa 520 X-ray microscope. When performing LabDCT the main (transmitted) beam of X-rays is blocked by a coupled aperture (placed on the source) and beam-stop (placed on the detector). The detector, placed in the Laue focusing position (such that the source-sample and sample-detector distances are equal), detects an X-ray diffraction pattern in the region of the detector outside of the beamstop, associated with the X-rays diffracted by the crystal lattices of the materials in the sample. The sample is then rotated, from which a $3 \mathrm{D}$ reconstruction of crystal geometry can be created.

Results on a set of disaggregated fragments from the San Carlos Olivine show that, while globally crystal orientations were isotropic (figure 1A), crystal misorientations in specific (spatially associated) subfragments were highly correlated, with crystal misorientations of only around 1 degree. These subcrystals were well-defined throughout the volume, clearly resolvable in 3D and survive bulk rock disaggregation well, potentially indicating that rock disaggregation is associated with macro-crystal (rather than sub-crystal) interfaces. The porphyritic olivine chondrule shows a radial distribution in crystal quality (Fig. 2A). Olivine grains in the central portion of the chondrule are larger and better defined while the those around the periphery are smaller with highly irregular boundaries, suggesting that they may be deformed. Possible explanations for deformed or irregular peripheral grains include impact deformation 
on the asteroid parent body or reactions with the surrounding nebular gas during chondrule formation [4]. The distribution of crystallographic orientations in the central region, which we are still analyzing, may reveal whether they nucleated independently or shared some common control. The barred olivine chondrule contained a higher proportion of other mineral components, which posed a significant challenge to DCT reconstruction, a mineralogical mask was created by segmenting a spatially correlated absorption tomography image, such that only regions associated with the olivine were examined in diffraction space (Fig. 2B-C). Such an approach may have wide application in the geosciences where multi-mineralogy systems are common, as recent developments in using machine learning based classification techniques have allowed for mineralogy to be quantified in $3 \mathrm{D}[5]$.

\section{References:}

[1] DJ Prior, E Mariani and Wheeler J. Mater. Sci. (2009).

[2] W Ludwig et al., Rev Sci Instrum (2009).

[3] SA McDonald et al., Sci Rep (2015).

[4] P Friend, DC Hezel and D Mucerschi, Geochim Cosmochim Acta 173 (2016), p. 198.

[5] M Andrew, Comput Geosci 22 (2018), p. 1503.

A

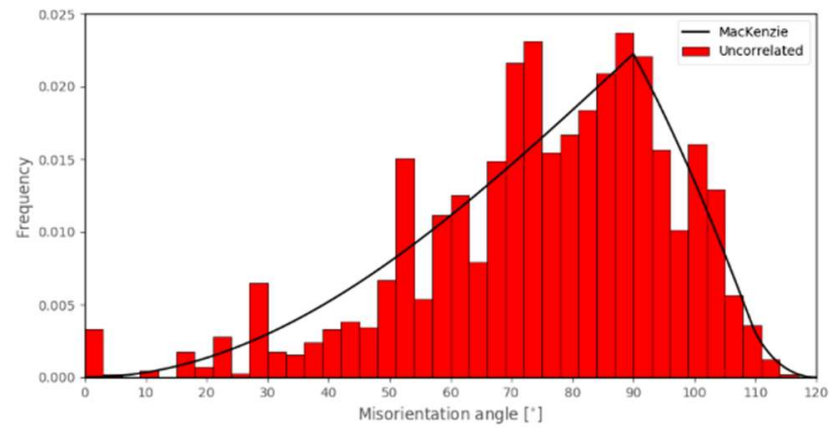

B

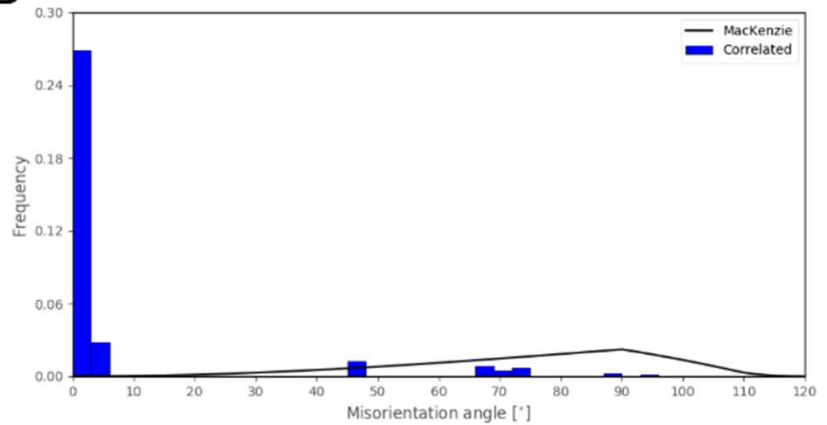

Figure 1. Crystal misorientation distributions for the global (uncorrelated) misorientation set (A) and the correlated misorientation set for spatially associated crystals (B) in the San Carlos olivine
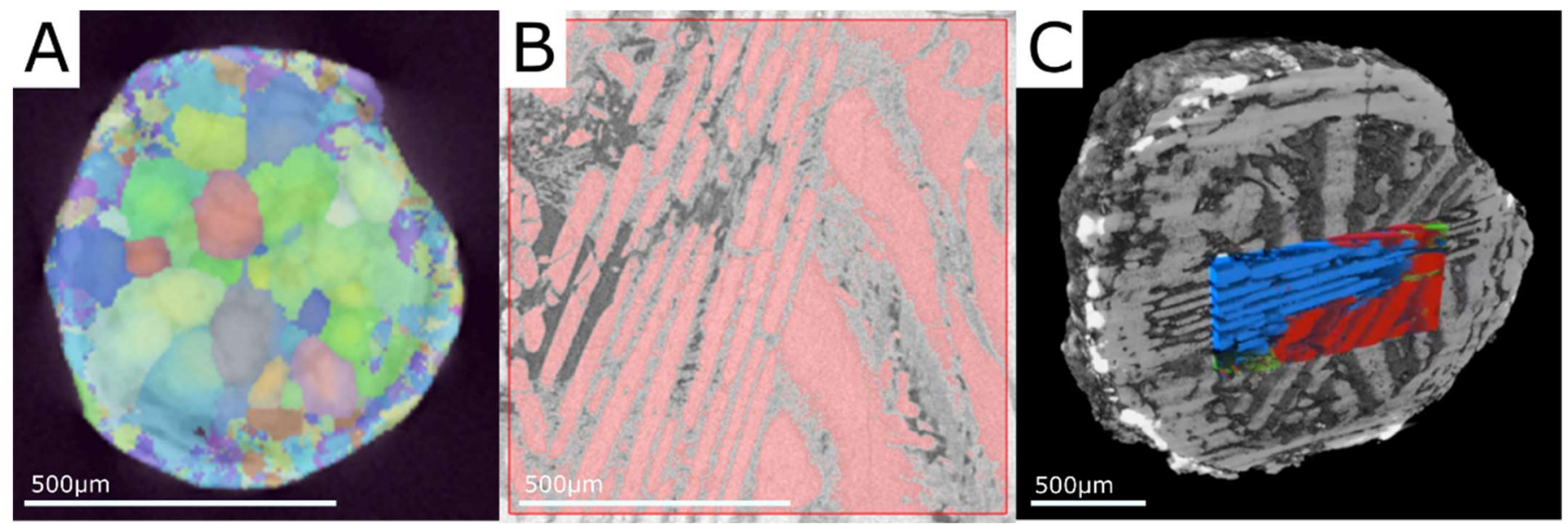

Figure 2. (A) Porphyritic olivine chondrule crystal orientations, showing well-formed crystals at the center of the chondrule and poorly-formed crystals towards the edge (grains are colored by Grain ID).

(B) Mineralogical mask (red), constructed using traditional (absorption contrast) tomography, used for the reconstruction of olivine crystal orientations (color) in the barred olivine chondrule. (C) 